\title{
Existence theorem and optimality conditions for a class of convex semi-infinite problems with noncompact index sets
}

\author{
Olga Kostyukova ${ }^{1}$, Tatiana Tchemisova ${ }^{2, *}$, Maryia Kurdina ${ }^{1}$ \\ ${ }^{1}$ Institute of Mathematics, National Academy of Sciences of Belarus, Minsk, Belarus \\ ${ }^{2}$ Center for Research and Development in Mathematics and Applications, University of Aveiro, Aveiro, Portugal
}

(Received: 23 August 2017; Accepted: 22 October 2017)

\begin{abstract}
The paper is devoted to study of a special class of semi-infinite problems arising in nonlinear parametric optimization. These semi-infinite problems are convex and possess noncompact index sets. In the paper, we present conditions, which guarantee the existence of optimal solutions, and prove new optimality criterion. An example illustrating the obtained results is presented.
\end{abstract}

Keywords Semi-Infinite Programming (SIP), Linear Programming (LP), Quadratic Programming (QP), Constraint Qualifications (CQ).

AMS 2010 subject classifications 65K10, 90C20, 90C25, 90C30, 90C34, 90C4.

DOI: $10.19139 /$ soic.v5i4.362

\section{Introduction}

Semi-infinite Programming (SIP) deals with optimization problems, which have an infinite number of constraints. SIP has always been a topic of a special interest due to the numerous theoretical and practical applications such as robotic, classical engineering, optimal design, the Chebyshev approximations, etc. (see [6, 7, 8], and the references therein). Nowadays, SIP models are efficiently used in dynamic processes, biomedical and chemical engineering, biology, tissue engineering, polymer reaction engineering, etc. (see [1, 16], and others). A general SIP problem can be formulated as

$$
\min _{\varkappa \in \mathbb{R}^{n}} c(\varkappa) \quad \text { s.t. } \quad f(\varkappa, \tau) \leq 0 \forall \tau \in T,
$$

where $\varkappa \in \mathbb{R}^{n}$ is a decision variable, $\tau$ is a constraint' index, $T \subset \mathbb{R}^{p}$ is an infinite index set. When, additionally, the index set $T$ depends on the decision variable $\varkappa$, one gets a problem of the generalized SIP (see [9]). We say that a SIP problem is continuous whenever the index set $T$ is a compact Hausdorff topological space and the functions $c(\varkappa)$ and $f(\varkappa, \tau)$ are continuous w.r.t. their variables. The compactness of the index set $T$ ensures the existence of global maximizers of the so-called lower level problem: $\max _{t \in T} f(\varkappa, \tau)$. The continuity of the functions defining the SIP problem is a natural condition, which permits to apply the methods of continuous optimization. Usually, it is assumed that a SIP problem is continuous. However, it should be noted that there are some classes of problems, for which noncompact index set is commonplace. Without the compactness of $T$ and/or the continuity of the inequality

\footnotetext{
*Correspondence to: Tatiana Tchemisova (Email: tatiana@ua.pt). Center for Research and Development in Mathematics and Applications, Department of Mathematics, University of Aveiro, Campus Universitário Santiago, 3810-193, Aveiro, Portugal.
}

ISSN 2310-5070 (online) ISSN 2311-004X (print)

Copyright (C) 2017 International Academic Press 
constraint function with respect to the index variable, the properties of SIP problems change dramatically and the known SIP theory and methods may fail.

In this paper, we explore a class of SIP problems, in which the corresponding index sets have a conic structure and hence are not compact. Such problems arise in nonlinear parametric SIP when the differential properties of the solutions are being studied, and therefore, it is important to study these problems, which is the main purpose of this paper.

The rest of the paper is organized as follows. In Section 2, we state an optimization problem arising in nonlinear parametric SIP, and formulate the main aims of the paper. In Section 3, we show that the optimization problem formulated in Section 2 can be reduced to a convex SIP problem of special form with noncompact index set. In Section 4 , for the special class of convex SIP problems with noncompact index sets, we present the existence theorem and discuss a possibility of its strengthening, and in Section 5, we formulate and prove the optimality conditions. Section 6 contains an example, which illustrates that the results obtained in the paper can be applied in situations, where the classical optimality results are not efficient due to noncompactness of the index set. The conclusions and final remarks are done in the final Section 7. In Appendix, we prove Proposition 1, which is need for the proof of the main results of the paper.

\section{Problem statement}

Let finite index sets $J \subset \mathbb{N}, S_{*} \subset \mathbb{N}$, and $S \subset \mathbb{N}$, such that $S_{*} \cap S=\emptyset$, as well as matrices, vectors and numbers

$$
\begin{gathered}
\bar{W}_{j} \in \mathbb{R}^{n \times n}, \bar{d}_{j} \in \mathbb{R}^{n}, \bar{r}_{j} \in \mathbb{R}, g_{j} \in \mathbb{R}^{n}, j \in J, \quad c \in \mathbb{R}^{n}, \\
W_{0} \in \mathbb{R}^{n \times n}, d_{0} \in \mathbb{R}^{n}, r_{0} \in \mathbb{R} ; \quad q_{j} \in \mathbb{R}^{n}, \omega_{j} \in \mathbb{R}, j \in S_{*} \cup S, \\
D_{j} \in \mathbb{R}^{p \times p}, A_{j} \in \mathbb{R}^{n \times p}, B_{j} \in \mathbb{R}^{m_{j} \times p}, c_{j} \in \mathbb{R}^{p}, j \in J,
\end{gathered}
$$

be given. Define the following sets:

$$
\begin{aligned}
& Y=\left\{y=\left(y_{j}, j \in J\right): \sum_{j \in J} g_{j} y_{j}=c, y_{j} \geq 0, j \in J\right\}, \\
& \mathcal{X}=\left\{x \in \mathbb{R}^{n}: q_{j}^{T} x+\omega_{j}=0, j \in S_{*}, \quad q_{j}^{T} x+\omega_{j} \leq 0, j \in S\right\}, \\
& K(j)=\left\{t \in \mathbb{R}^{p}: B_{j} t \leq 0\right\}, \quad j \in J,
\end{aligned}
$$

and suppose that

1) the (polyhedral) set $Y$ is nonempty and bounded;

2) the set $\mathcal{X}$ is nonempty;

3) the following inequalities are satisfied:

$$
x^{T} W_{0} x \geq 0, x^{T} \bar{W}_{j} x \geq 0, \forall x \in \mathbb{R}^{n}, t^{T} D_{j} t \geq 0 \forall t \in K(j), j \in J .
$$

Consider the following optimization problem:

$$
\min _{x \in \mathcal{X}}\left[\Omega_{0}(x)+\max _{y \in Y} \sum_{j \in J} y_{j}\left(\Omega_{j}(x)-\min _{t \in K(j)} \Psi_{j}(x, t)\right)\right],
$$

where

$$
\begin{gathered}
\Omega_{0}(x):=\frac{1}{2} x^{T} W_{0} x+d_{0}^{T} x+r_{0}, \quad \Omega_{j}(x):=\frac{1}{2} x^{T} \bar{W}_{j} x+\bar{d}_{j}^{T} x+\bar{r}_{j}, j \in J ; \\
\Psi_{j}(x, t):=\frac{1}{2} t^{T} D_{j} t+\left(c_{j}^{T}-x^{T} A_{j}\right) t, j \in J .
\end{gathered}
$$


Note that in (1), functions $\Omega_{j}(x), j \in J \cup\{0\}$, are convex w.r.t. $x \in \mathbb{R}^{n}$, and functions $\Psi_{j}(x, t), j \in J$, are linear w.r.t. $x \in \mathbb{R}^{n}$, and, in general, are non-convex w.r.t. $t \in K(j), j \in J$.

The problems in the form (1) arise in nonlinear parametric SIP when the differential properties of solutions are being studied $[10,12]$. Therefore, it is important to study the properties of these optimization problems.

The main aims of this paper are as follows:

- to show that the optimization problem (1) is equivalent to some special SIP problem;

- to study the issues connected with conditions, which guarantee the existence of optimal solutions of the special SIP problem (it should be mentioned that, at the moment, such conditions are not very well studied in SIP);

- to formulate the optimality conditions for the special SIP problem.

\section{Semi-infinite formulation}

Let $y^{(i)}=\left(y_{j}^{(i)}, j \in J\right), i \in I$, be the vertices (the extremal points) of the polyhedral set $Y$ defined in Section 2. In what follows, we suppose that these vertices are known.

Let us show that

$$
\max _{y \in Y} y^{T} f=\max _{i \in I} y^{(i)^{T}} f
$$

for any $f \in \mathbb{R}^{|J|}$.

In fact, since $y^{(i)} \in Y, i \in I$, then $\max _{y \in Y} y^{T} f \geq \max _{i \in I} y^{(i)^{T}} f$. Suppose that

$$
\max _{y \in Y} y^{T} f=y^{0^{T}} f>\max _{i \in I} y^{(i)^{T}} f
$$

where $y^{0} \in Y$. Consequently,

$$
y^{0^{T}} f>y^{(i)^{T}} f, \quad i \in I .
$$

Since $y^{0} \in Y$, then there exist numbers $\lambda_{i} \geq 0, i \in I, \sum_{i \in I} \lambda_{i}=1$, such that $y^{0}=\sum_{i \in I} \lambda_{i} y^{(i)}$.

Hence, $y^{0^{T}} f=\sum_{i \in I} \lambda_{i} y^{(i)^{T}} f$. From the last equality and (4), it follows that

$$
y^{0^{T}} f=\sum_{i \in I} \lambda_{i} y^{(i)^{T}} f<\sum_{i \in I} \lambda_{i} y^{0^{T}} f=y^{0^{T}} f .
$$

The resulting contradiction proves that (3) is false, and hence (2) holds.

Taking into account equality (2), we conclude that the optimization problem (1) is equivalent to the following one:

$$
\min _{x \in \mathcal{X}}\left[\Omega_{0}(x)+\max _{i \in I} \sum_{j \in J} y_{j}^{(i)}\left(\Omega_{j}(x)-\min _{t \in K(j)} \Psi_{j}(x, t)\right)\right] .
$$

Denote $W_{i}:=\sum_{j \in J} y_{j}^{(i)} \bar{W}_{j}, \quad d_{i}:=\sum_{j \in J} y_{j}^{(i)} \bar{d}_{j}, \quad r_{i}:=\sum_{j \in J} y_{j}^{(i)} \bar{r}_{j}, \quad i \in I$, and

$$
\rho_{j}(x):=\min _{t \in K(j)}\left(\frac{1}{2} t^{T} D_{j} t+\left(c_{j}^{T}-x^{T} A_{j}\right) t\right), j \in J .
$$

Then, problem (5) can be rewritten in the form

$$
\begin{array}{ll} 
& \min _{x, \beta} \frac{1}{2} x^{T} W_{0} x+d_{0}^{T} x+\beta \\
\text { s.t. } & \frac{1}{2} x^{T} W_{i} x+d_{i}^{T} x+r_{i}-\sum_{j \in J} y_{j}^{(i)} \rho_{j}(x) \leq \beta, i \in I ; \quad x \in \mathcal{X} .
\end{array}
$$


Taking into account that $y_{j}^{(i)} \geq 0, i \in I, j \in J$, one can show that the last problem is equivalent to the following SIP problem:

$$
\begin{array}{ll} 
& \min _{x, \rho_{j}, j \in J, \beta} \quad \frac{1}{2} x^{T} W_{0} x+d_{0}^{T} x+\beta \\
\text { s.t. } & \frac{1}{2} x^{T} W_{i} x+d_{i}^{T} x+r_{i}-\sum_{j \in J} y_{j}^{(i)} \rho_{j} \leq \beta, i \in I ; \quad x \in \mathcal{X}, \\
& \rho_{j} \leq \frac{1}{2} t_{j}^{T} D_{j} t_{j}+\left(c_{j}^{T}-x^{T} A_{j}\right) t_{j}, \quad \forall t_{j} \in K(j), \quad j \in J .
\end{array}
$$

In problem (7), the decision variables form the vector $\phi=\left(x \in \mathbb{R}^{n}, \rho_{j} \in \mathbb{R}, j \in J, \beta \in \mathbb{R}\right)$. Without loss of generality, we may suppose that

$$
\sum_{i \in I} y_{j}^{(i)}>0, j \in J ; \sum_{j \in J} y_{j}^{(i)}>0, i \in I
$$

Let us make some observations concerning problem (7).

- The constraints and the cost function of this problem are linear-quadratic w.r.t. decision variables $x \in$ $\mathbb{R}^{n}, \rho_{j} \in \mathbb{R}, j \in J$, and $\beta$. Hence, it is evident that this problem is convex, and each its local optimal solution is a global one.

- If problem (7) is consistent, then its linear constraints (infinite number)

$$
-\frac{1}{2} t_{j}^{T} D_{j} t_{j}-\left(c_{j}-A_{j}^{T} x\right)^{T} t_{j}+\rho_{j} \leq 0, \quad \forall t_{j} \in K(j), j \in J,
$$

and linear-quadratic constraints (finite number)

$$
x^{T} W_{i} x+d_{i}^{T} x+r_{i}-\sum_{j \in J} y_{j}^{(i)} \rho_{j}-\beta \leq 0, i \in I
$$

satisfy the Slater condition (i.e. these constraints are strictly satisfied for some $\bar{x} \in \mathcal{X}, \bar{\rho} \in \mathbb{R}^{|J|}, \bar{\beta} \in \mathbb{R}$ ).

- Since $t_{j}=0 \in K(j)$, it is evident that

$$
\rho_{j} \leq 0, j \in J
$$

- In problem (7), the index sets $K(j), j \in J$, are not compacts.

- For any feasible solution $\left(x, \rho_{j}, j \in J, \beta\right)$, the following relations take place:

$$
\left(c_{j}^{T}-x^{T} A_{j}\right) \tau_{j} \geq 0 \forall \tau_{j} \in \Delta K(j):=\left\{\tau \in K(j): \tau^{T} D_{j} \tau=0\right\}, j \in J .
$$

Let us define the function

$$
\beta(x):=\max _{i \in I}\left(\frac{1}{2} x^{T} W_{j} x+d_{j}^{T} x+r_{j}-\sum_{j \in J} y_{j}^{(i)} \rho_{j}(x)\right) .
$$

Note that for any feasible solution $\left(x, \rho_{j}, j \in J, \beta\right)$ of problem (7), there exists a feasible solution

$$
\left(x, \rho_{j}(x), j \in J, \beta(x)\right),
$$

such that $\beta(x)$ satisfies $(11)$ and $\beta(x) \leq \beta$. Hence, without loss of generality, in what follows, we can consider only feasible solutions in the form (12).

As it was revealed above, our interest to the problems in the form (7) arose from the study of the solutions' properties in parametric SIP problems w.r.t. perturbations of parameters. Though, it is worth mentioning that problem (7) is an interesting subject itself. Similar SIP problems were considered, for example, in [3, 5], et al. The references that one can find in these papers, indicate also on other areas, where such problems appear. 


\section{On existence of optimal solutions of problem (7)}

\subsection{Sufficient conditions guaranteeing existence of optimal solutions}

The main result of this section is Theorem 1, which gives a sufficient conditions of solvability of the convex SIP problem (7).

Given problem (7), consider the set

$$
\begin{gathered}
\Delta X=\left\{\Delta x \in \mathbb{R}^{n}: q_{j}^{T} \Delta x=0, j \in S_{*} ; q_{j}^{T} \Delta x \leq 0, j \in S ; W_{i} \Delta x=0, i \in I \cup\{0\} ;\right. \\
\left.\exists \mu^{*}(j)=\mu^{*}(\Delta x, j) \geq 0 \text { such that } \Delta x^{T} A_{j}=\mu^{* T}(j) B_{j}, j \in J\right\} .
\end{gathered}
$$

\section{Theorem 1}

Suppose that

(A) there exists $\bar{x} \in \mathcal{X}$ such that

$$
\left(c_{j}^{T}-\bar{x}^{T} A_{j}\right) \tau_{j} \geq 0 \forall \tau_{j} \in \Delta K(j), j \in J,
$$

(B) either the set $\Delta X \backslash\{0\}$ is empty or the following implication takes place:

$$
\Delta x \in \Delta X \backslash\{0\} \Rightarrow d_{0}^{T} \Delta x+\max _{i \in I} d_{i}^{T} \Delta x>0 .
$$

Then problem (7) has an optimal solution.

Proof. Let us prove, first, that under condition (A), the feasible set $X$ in problem (7) is nonempty. Note that it follows from assumption 2) (see Section 2) and condition (A), that $\mathcal{X} \neq \emptyset$ and there exists $\bar{x} \in \mathcal{X}$ satisfying (14).

Given $\bar{x} \in \mathcal{X}$ and $j \in J$, consider a Quadratic Programming (QP) problem

$$
\rho_{j}(\bar{x})=\min _{t \in K(j)}\left(\frac{1}{2} t^{T} D_{j} t+c_{j}^{T} t-\bar{x}^{T} A_{j} t\right) .
$$

Taking into account condition (A) and the results from [4], we conclude that the problem above has an optimal solution. Consequently, the vector $\left(\bar{x}, \rho_{j}(\bar{x}), j \in J, \beta(\bar{x})\right)$ (with $\beta(x)$ defined in (11)) is a feasible solution of problem (7) and the set of feasible solutions $X$ in this problem is nonempty. As it was noted in Section 3, for any $\left(x, \rho_{j}, j \in J, \beta\right) \in X$, there exists a feasible solution $\left(x, \rho_{j}(x), j \in J, \beta(x)\right)$, such that $\beta(x) \leq \beta$. Hence, without loss of generality, we can consider only such feasible solutions.

Now, let us prove that, if, additionally, the condition (B) is satisfied, then the convex problem (7) has an optimal solution. Consider any sequence of feasible solutions $x^{k} \in X, k=1,2, \ldots$, such that the corresponding sequence of the cost function values of problem (7) decreases. Hence the following inequalities take place:

$$
x^{k^{T}} W_{0} x^{k}+d_{0}^{T} x^{k}+\beta\left(x^{k}\right) \leq x^{1^{T}} W_{0} x^{1}+d_{0}^{T} x^{1}+\beta\left(x^{1}\right)=\text { const }, \quad k=1,2 \ldots
$$

Let us show that there exists a number $M_{0}>0$ such that $\left\|x^{k}\right\| \leq M_{0}, k=1,2, \ldots$ Having supposed that on the contrary, such $M_{0}$ does not exist, without loss of generality, we can consider that $\left\|x^{k}\right\| \rightarrow \infty$ as $k \rightarrow \infty$. It follows from the constraints of (7) that

$$
\frac{1}{2} x^{k T} W_{i} x^{k}+d_{i}^{T} x^{k}+r_{i}-\sum_{j \in J} y_{j}^{(i)} \rho_{j}\left(x^{k}\right) \leq \beta\left(x^{k}\right), i \in I .
$$

Divide inequalities (15) and (16) by $\left\|x^{k}\right\|^{2}$, and pass to the limit as $k \rightarrow \infty$. As a result, we obtain

$$
\Delta x^{* T} W_{0} \Delta x^{*}+\lim _{k \rightarrow \infty} \frac{\beta\left(x^{k}\right)}{\left\|x^{k}\right\|^{2}} \leq 0, \frac{1}{2} \Delta x^{* T} W_{i} \Delta x^{*}-\sum_{j \in J} y_{j}^{(i)} \Delta \rho_{j}^{*} \leq \lim _{k \rightarrow \infty} \frac{\beta\left(x^{k}\right)}{\left\|x^{k}\right\|^{2}}, i \in I,
$$


where $\Delta x^{*}=\lim _{k \rightarrow \infty} \frac{x^{k}}{\left\|x^{k}\right\|}, \Delta \rho_{j}^{*}=\lim _{k \rightarrow \infty} \frac{\rho_{j}\left(x^{k}\right)}{\left\|x^{k}\right\|^{2}}, j \in J$.

Taking into account inequalities (8), (9), and the positive semi-definitiveness of the matrices $W_{i}, i \in I \cup\{0\}$, we conclude from (17) that

$$
W_{i} \Delta x^{*}=0, i \in I \cup\{0\} ; \Delta \rho_{j}^{*}=0, j \in J .
$$

Moreover, it is easy to show that

$$
q_{j}^{T} \Delta x^{*}=0, j \in S_{*} ; q_{j}^{T} \Delta x^{*} \leq 0, j \in S .
$$

For the fixed $j \in J$ and $k \in \mathbb{N}$, consider the problem

$$
\min _{t \in K(j)}\left(\frac{1}{2} t^{T} D_{j} t+c_{j}^{T} t-x^{k^{T}} A_{j} t\right)
$$

Since $x^{k} \in X$, this problem has an optimal solution, which we denote here by $t_{j}^{k} \in K(j), \rho_{j}\left(x^{k}\right)$ being the optimal value of the cost function. Without loss of generality, let us consider that $t_{j}^{k}$ is an optimal solution, which has the minimal norm. For $t_{j}^{k}$, the following first order (necessary) optimality conditions take place:

$$
D_{j} t_{j}^{k}+c_{j}-A_{j}^{T} x^{k}+B_{j}^{T} \mu(k, j)=0, \mu(k, j) \geq 0, t_{j}^{k^{T}} B_{j}^{T} \mu(k, j)=0 .
$$

Below, with no loss of generality, we will suppose that in (21) it holds $\mu(k, j)=\mu^{*}(k, j)$ with

$$
\mu^{*}(k, j):=\arg \min _{\mu \in \mathcal{M}(k, j)}\|\mu\|
$$

where $\mathcal{M}(k, j) \subset \mathbb{R}^{m_{j}}$ is the set of all $\mu(k, j)$ satisfying (21). It follows from (21), that

$$
\rho_{j}\left(x^{k}\right)=-\frac{1}{2} t_{j}^{k^{T}} D_{j} t_{j}^{k}
$$

Having divided both sides of this equality by $\left\|x^{k}\right\|^{2}$ and passing to the limit as $k \rightarrow \infty$, we get

$$
0=\Delta \rho_{j}^{*}=-\frac{1}{2} \Delta t_{j}^{T} D_{j} \Delta t_{j} \sigma^{2} \text { with } \Delta t_{j}=\lim _{k \rightarrow \infty} \frac{t_{j}^{k}}{\left\|t_{j}^{k}\right\|}, \sigma=\lim _{k \rightarrow \infty} \frac{\left\|t_{j}^{k}\right\|}{\left\|x^{k}\right\|} .
$$

There are two possible situations here: I) $\sigma>0, \Delta t_{j}^{T} D_{j} \Delta t_{j}=0$, and $\quad$ II) $\sigma=0$.

Suppose, first, that the situation I) takes place. Hence, there exists $\Delta t_{j}$ such that

$$
\Delta t_{j} \neq 0, \Delta t_{j}^{T} D_{j} \Delta t_{j}=0, B_{j} \Delta t_{j} \leq 0 .
$$

Denote

$$
M_{a}^{k}(j):=\left\{m \in\left\{1, \ldots, m_{j}\right\}: b_{m j}^{T} t_{j}^{k}=0\right\}, M_{a}(j):=\left\{m \in\left\{1, \ldots, m_{j}\right\}: b_{m j}^{T} \Delta t_{j}=0\right\} .
$$

Here and in what follows, $b_{m j}^{T}$ denotes the $m$-th row of matrix $B_{j}$.

For sufficiently large $k$, it is evident that $M_{a}^{k}(j) \subset M_{a}(j)$ and from (21) we conclude that $\mu_{m}(k, j)=0$, $m \in\left\{1, \ldots, m_{j}\right\} \backslash M_{a}^{k}(j)$. Here $\mu_{m}(k, j)$ denotes the $m$-th element of vector $\mu(k, j) \in \mathbb{R}^{m_{j}}$. Based on these observations, it is easy to show that $(\mu(k, j))^{T} B_{j} \Delta t_{j}=0$ for sufficiently large $k$. Taking into account this equality, let us multiply the first equality in (21) by $\Delta t_{j}^{T}$ :

$$
\Delta t_{j}^{T} D_{j} t_{j}^{k}+\left(c_{j}^{T}-x^{k^{T}} A_{j}\right) \Delta t_{j}=0 .
$$

If suppose that $\left(c_{j}^{T}-x^{k^{T}} A_{j}\right) \Delta t_{j}<0$, then we should conclude that the cost function of problem (20) in not bounded from below on the feasible set. But this is impossible, since this problem admits an optimal solution. 
Therefore, the following inequality holds true:

$$
\left(c_{j}^{T}-x^{k^{T}} A_{j}\right) \Delta t_{j} \geq 0 .
$$

Let us show that for any $j \in J$,

$$
t^{T} D_{j} \tau \geq 0 \text { for all } t \in K(j), \tau \in \Delta K(j) .
$$

In fact, for any $t \in K(j), \tau \in \Delta K(j)$ and $\theta \geq 0$, we have $(\theta t+\tau) \in K(j)$. Hence for all $\theta \geq 0$ it holds $0 \leq(\theta t+\tau)^{T} D_{j}(\theta t+\tau)=\theta^{2} t^{T} D_{j} t+\theta t^{T} D_{j} \tau$. For a sufficiently small $\theta \geq 0$, it follows from the last inequality that $t^{T} D_{j} \tau \geq 0$ and the relations (25) are proved.

Since $t_{j}^{k} \in K(j), \Delta t_{j}^{T} D_{j} \Delta t_{j}=0, \Delta t_{j} \in \Delta K(j)$, it follows from (25) that $\Delta t_{j}^{T} D_{j} t_{j}^{k} \geq 0$. Then the last inequality together with (23) and (24) imply

$$
\left(c_{j}^{T}-x^{k^{T}} A_{j}\right) \Delta t_{j}=0, \Delta t_{j}^{T} D_{j} t_{j}^{k}=0 .
$$

By construction, the vector $t_{j}^{k}$ can be written in the form

$$
t_{j}^{k}=\left(\Delta t_{j}+\varepsilon_{k}\right) \tilde{\theta}_{k}, \text { where } \tilde{\theta}_{k}=\left\|t_{j}^{k}\right\|, \varepsilon_{k} \rightarrow 0 \text { as } k \rightarrow \infty .
$$

Taking into account this representation, we conclude from (26) that

$$
0=\Delta t_{j}^{T} D_{j}\left(\Delta t_{j}+\varepsilon_{k}\right) \tilde{\theta}_{k} \Rightarrow \Delta t_{j}^{T} D_{j} \varepsilon_{k}=0, k=1,2, \ldots,
$$

and from the inequalities $b_{m j}^{T} t_{j}^{k} \leq 0, m=1, \ldots, m_{j}$, we get $b_{m j}^{T}\left(\Delta t_{j}+\varepsilon_{k}\right) \leq 0, m=1, \ldots, m_{j}$. Consequently, for $m=1, \ldots, m_{j}$, the following implication is valid: if $b_{m j}^{T} \varepsilon_{k}>0$, then $b_{m j}^{T} \Delta t_{j}<0$.

Let us set $\tilde{\alpha}_{m}(k)=0$ if $b_{m j}^{T} \varepsilon_{k} \leq 0, \tilde{\alpha}_{m}(k)=-\frac{b_{m j}^{T} \varepsilon_{k}}{b_{m j}^{T} \Delta t_{j}}$ if $b_{m j}^{T} \varepsilon_{k}>0, m=1, \ldots, m_{j}$, and calculate

$$
\tilde{\alpha}(k):=\max \left\{\tilde{\alpha}_{m}(k), m=1, \ldots, m_{j}\right\} .
$$

It is easy to check that $\tilde{\alpha}(k) \rightarrow 0$ as $k \rightarrow \infty$. Consider vector $\tilde{t}_{j}^{k}:=\left(\tilde{\alpha}(k) \Delta t_{j}+\varepsilon_{k}\right) \tilde{\theta}_{k}$. By construction, it holds

$$
B_{j} \tilde{t}_{j}^{k} \leq 0 \Leftrightarrow \tilde{t}_{j}^{k} \in K(j)
$$

Taking into account (26)-(28), we get

$$
\begin{gathered}
\tilde{t}_{j}^{k T} D_{j} \tilde{t}_{j}^{k}=\tilde{\theta}_{k}^{2} \varepsilon_{k}^{T} D_{j} \varepsilon_{k}, \quad t_{j}^{k T} D_{j} t_{j}^{k}=\tilde{\theta}_{k}^{2} \varepsilon_{k}^{T} D_{j} \varepsilon_{k}, \\
\left(c_{j}^{T}-x^{k T} A_{j}\right) \tilde{t}_{j}^{k}=\tilde{\theta}_{k}\left(c_{j}^{T}-x^{k T} A_{j}\right) \varepsilon_{k}, \quad\left(c_{j}^{T}-x^{k T} A_{j}\right) t_{j}^{k}=\tilde{\theta}_{k}\left(c_{j}^{T}-x^{k T} A_{j}\right) \varepsilon_{k} .
\end{gathered}
$$

From the last equalities, it follows that for $k=1,2, \ldots$, both vectors, $\tilde{t}_{j}^{k}$ and $t_{j}^{k}$ are optimal solutions of problem (20). Note that for the large numbers $k$, the inequality $\mid \tilde{t}_{j}^{k}\|<\| t_{j}^{k} \|$ takes place, that is impossible since $t_{j}^{k}$ is the minimal norm optimal solution of problem (20). The obtained contradiction permits to conclude that the situation I) is not possible.

Now, suppose that the situation II) takes a place:

$$
\sigma=\lim _{k \rightarrow \infty} \frac{\left\|t_{j}^{k}\right\|}{\left\|x^{k}\right\|}=0
$$

Let us show that $\lim _{k \rightarrow \infty} \frac{\|\mu(k, j)\|}{\left\|x^{k}\right\|}<\infty$ for $\mu(k, j)$ defined in (21). Having supposed that, on the contrary, $\lim _{k \rightarrow \infty} \frac{\|\mu(k, j)\|}{\left\|x^{k}\right\|}=\infty$, we get $\lim _{k \rightarrow \infty} \frac{\left\|x^{k}\right\|}{\|\mu(k, j)\|}=0$. Taking into account the last equality and (29), let us divide all 
relations in (21) by $\|\mu(k, j)\|$ and pass to the limit as $k \rightarrow \infty$. As a result, we obtain

$$
B^{T} \Delta \mu(j)=0, \Delta \mu(j) \geq 0,\|\Delta \mu(j)\|=1 \text { with } \Delta \mu(j)=\lim _{k \rightarrow \infty} \frac{\mu(k, j)}{\|\mu(k, j)\|} .
$$

Hence, vector $\mu(k, j)$ admits representation $\mu(k, j)=(\Delta \mu(j)+w(k, j)) \theta_{k}$, where $\theta_{k}=\|\mu(k, j)\|, w(k, j) \rightarrow 0$ as $k \rightarrow \infty$. The relations (21) can be rewritten in the form

$$
D_{j} t_{j}^{k}+c_{j}-A_{j}^{T} x^{k}+B_{j}^{T} w(k, j) \theta_{k}=0, \Delta \mu(j)+w(k, j) \geq 0, t_{j}^{k T} B_{j}^{T} w(k, j)=0, k=1,2, \ldots
$$

Let $\Delta \mu_{i}(j), w_{i}(k, j)$ be the $i$-th components of the vectors $\Delta \mu(j), w(k, j), i=1, \ldots, m_{j}$. From (30), we conclude that given $i \in\left\{1, \ldots, m_{j}\right\}$, the inequality $w_{i}(k, j)<0$ implies $\Delta \mu_{i}(j)>0$.

Let us calculate $\alpha(k)=\max \left\{\alpha_{i}(k), i=1, \ldots m_{j}\right\}$, where

$$
\alpha_{i}(k)=0 \text { if } w_{i}(k, j) \geq 0 \text {, and } \alpha_{i}(k)=-w_{i}(k, j) / \Delta \mu_{i}(j) \text { if } w_{i}(k, j)<0, i=1, \ldots, m_{j} .
$$

It is evident that $\alpha(k) \geq 0, \lim _{k \rightarrow \infty} \alpha(k)=0$, and, by construction, for $\bar{\mu}(k, j):=\theta_{k}(\alpha(k) \Delta \mu(j)+w(k, j))$ we have

$$
0=D_{j} t_{j}^{k}+c_{j}-A_{j}^{T} x^{k}+B_{j}^{T} \bar{\mu}(k, j), \bar{\mu}(k, j) \geq 0,0=t_{j}^{k T} B_{j}^{T} \bar{\mu}(k, j), k=1,2, \ldots
$$

Hence, $\bar{\mu}(k, j) \in \mathcal{M}(k, j)$ and $\|\bar{\mu}(k, j)\|=\|\mu(k, j)\| \cdot\|(\alpha(k) \Delta \mu(j)+w(k, j))\|$.

Note that $\|(\alpha(k) \Delta \mu(j)+w(k, j))\| \rightarrow 0$ as $k \rightarrow \infty$. Then, for sufficiently large $k$, we have $\|\bar{\mu}(k, j)\|<$ $\|\mu(k, j)\|$, that contradicts (22). Therefore, $\lim _{k \rightarrow \infty} \frac{\|\mu(k, j)\|}{\left\|x^{k}\right\|}<\infty$.

Now divide both sides of (21) by $\left\|x^{k}\right\|$ and pass to the limit as $k \rightarrow \infty$, taking into account (29). As a result, we obtain

$$
-A_{j}^{T} \Delta x^{*}+B_{j}^{T} \Delta \mu_{j}^{*}=0, \Delta \mu_{j}^{*} \geq 0 \text { with } \Delta \mu_{j}^{*}=\lim _{k \rightarrow \infty} \frac{\mu(k, j)}{\left\|x^{k}\right\|} .
$$

The last relations together with (18), (19) permit to conclude that $\Delta x^{*} \in \Delta X$.

It follows from (15) and (16) that $d_{0}^{T} x^{k}+\beta\left(x^{k}\right) \leq$ const, $d_{i}^{T} x^{k}+r_{i} \leq \beta\left(x^{k}\right), i \in I$. Hence

$$
d_{0}^{T} x^{k}+\max _{i \in I}\left(d_{i}^{T} x^{k}+r_{i}\right) \leq \text { const. }
$$

Divide both sides of the last inequality by $\left\|x^{k}\right\|$ and pass to the limit as $k \rightarrow \infty$. As a result, we get inequality $d_{0}^{T} \Delta x^{*}+d_{i}^{T} \Delta x^{*} \leq 0, i \in I$, which contradicts the assumption (B) of the theorem. Hence situation II) is impossible as well as situation I).

The obtained contradictions lead us to conclude that for any sequence of feasible solutions $x^{k} \in X, k=1,2, \ldots$, of problem (7), where $x^{k}$ satisfy inequalities (15), there exists $M_{0}>0$ such that $\left\|x^{k}\right\| \leq M_{0}, k=1,2, \ldots$ This fact permits to conclude that problem (7) admits an optimal solution. The theorem is proved.

At the end of this section, we would like to make the following remarks.

1. It can be shown that the feasible set $X$ of problem (7) is not empty if and only if the condition (A) of Theorem 1 is satisfied, that, in turn, always happens when $t_{j}^{T} D_{j} t_{j}>0$ for all $t_{j} \in K(j) \backslash\{0\}, j \in J$.

2. Note that the condition (B) is considered to be satisfied if $\Delta X \backslash\{0\}=\emptyset$. Hence, from (13), one can see that the condition (B) holds true, if the matrix $W:=\sum_{i \in I \cup\{0\}} W_{i}$ is (strictly) positive definite on the set

$$
\begin{gathered}
\Delta \tilde{X}:=\left\{\Delta x \in \mathbb{R}^{n}: q_{j}^{T} \Delta x=0, j \in S_{*} ; \quad q_{j}^{T} \Delta x \leq 0, j \in S ;\right. \\
\left.\exists \mu^{*}(j)=\mu^{*}(\Delta x, j) \geq 0, \text { such that } \Delta x^{T} A_{j}=\mu(j)^{* T} B_{j}, j \in J\right\} .
\end{gathered}
$$

This condition will be satisfied if at least one of the matrices $W_{i}, i \in I \cup\{0\}$, is positive definite on the set $\Delta \tilde{X}$. 
3. The implication

$$
\Delta x \in \Delta X \Rightarrow d_{0}^{T} \Delta x+\max _{i \in I} d_{i}^{T} \Delta x \geq 0,
$$

which is a slight modification of the condition (B), is a necessary condition for boundedness from below of the cost function in problem (7).

\subsection{Relaxation of the sufficient condition}

From the remarks done at the end of the previous subsection, one can conclude that the sufficient conditions formulated in Theorem 1, are also "almost" necessary for existence of an optimal solution in the convex SIP problem (7). The differences between the necessary and sufficient conditions appear only in the case, when there exists a vector $\Delta x \neq 0$ such that $\Delta x \in \Delta X, d_{0}^{T} \Delta x+\max _{i \in I} d_{i}^{T} \Delta x=0$.

The following conjecture of more strong statement naturally arises from our considerations.

Conjecture. Problem (7) admits an optimal solution if conditions (A) and (31) (a relaxed condition (B)) are satisfied.

At the moment, we have no proof of this statement and also have certain doubts about its truthfulness. The following example indicates that, possibly, the proposed conjecture is not true.

Example. Consider a linear SIP problem

$$
\min _{z} c^{T} z \quad \text { s.t. } a(t)^{T} z+b(t) \leq 0, \forall t \in T .
$$

Problem (7) has the form (32), if we set:

$$
\begin{gathered}
z^{T}=\left(x^{T}, \rho\right) \in \mathbb{R}^{n+1}, J=\{1\}, I=\{1\}, d_{0}=0, c^{T}=\left(d_{0}^{T},-y_{1}^{(1)}\right), \\
W_{0}=\mathbb{O}, W_{1}=\mathbb{O}, d_{1}=0, r_{1}=0, \mathcal{X}=\mathbb{R}^{n}, K(1)=T \\
a^{T}(t)=\left(t^{T} A_{1}^{T}, 1\right), b(t)=-\frac{1}{2} t^{T} D_{1} t-c_{1}^{T} t
\end{gathered}
$$

where $\mathbb{O}$ denotes the $n \times n$ null matrix.

For problem (32), the conditions (A) and (31) take the form of the following two conditions:

(A1) the constraints of problem (32) are consistent;

(B1) for any $\Delta z \in\left\{\Delta z \in \mathbb{R}^{n+1}: a(t)^{T} \Delta z \leq 0, \forall t \in T\right\}$, it holds $c^{T} \Delta z \geq 0$.

The conditions (A1) and (B1), in general, do not guarantee the existence of optimal solutions in problem (32) even if the index set $T$ is compact and the cost function of this problem is bounded in the feasible set. Indeed, let us consider example 5.101. from [2]:

$$
\min _{z} \bar{c}^{T} z=z_{2} \text { s.t. } \bar{a}(t)^{T} z+\bar{b}(t)=-t^{2} z_{1}-z_{2}+2 t \leq 0, \forall t \in T=[0,1] .
$$

The condition (A1) is satisfied here, since for any $k \geq 1$ vector $z^{k}=(k, 1 / k)^{T}$ is a feasible solution of this problem. The condition (B1) is fulfilled as well. Nevertheless, problem (33) does not have an optimal solution. Notice that there exists vector $\Delta \bar{z}=(1,0)^{T}$, such that

$$
\Delta \bar{z} \in\left\{\Delta z \in \mathbb{R}^{2}: \bar{a}(t)^{T} \Delta z \leq 0, \forall t \in T\right\} \text { and } \bar{c}^{T} \Delta \bar{z}=0,
$$

and the vector $z^{k}$ admits representation $z^{k}=(k, 1 / k)^{T}=k\left(\Delta \bar{z}+\omega_{k}\right)^{T}$, where $\omega_{k}=\left(0,1 / k^{2}\right)^{T} \rightarrow(0,0)^{T}$ as $k \rightarrow \infty$. 


\section{Optimality conditions for problem (7)}

The aim of this section is to formulate and prove optimality conditions for a given feasible solution of problem (7). It was mentioned in the Introduction, that in the majority of papers on optimality conditions for SIP problems, the compactness of the (infinite) index set of the (continual) constraints is being assumed. In our study of problem (7), this assumption is not satisfied: it was already noticed above that the sets $K(j), j \in J$, of the indices, which correspond to the continual constraints, are not compact.

In few papers dedicated to study of optimality for SIP problems without the assumption of the compactness of the index set (see, for example, [3, 5, 13], and references therein), it is supposed that the Farkas-Minkowski Constraint Qualification (CQ) is satisfied. In this paper, we do not have such an assumption for our problem (7) (see Theorem below).

In the literature, there is an approach, a so called technique of homogenization, [15], which also permits to bypass the difficulties caused by non-compactness of the index set. Having applied this approach to our problem, we obtain an equivalent SIP problem, which has a compact index set but does not satisfy the Slater condition: if $\exists j_{0} \in J, \Delta K\left(j_{0}\right) \neq \emptyset$, this new problem has immobile indices with infinite immobility orders [11]. Therefore, the known from the SIP literature necessary optimality conditions (see $[2,11,14]$ ) cannot be applied here too. Despite the above, in this section, for problem (7) with noncompact index set, we will prove the optimality criterion without any additional condition (CQ) on the constraints.

Let $\left(x, \rho_{j}, j \in J, \beta\right)$ be a feasible solution in problem (7). As it was mentioned above, without loss of generality, we can consider that $\left(x, \rho_{j}, j \in J, \beta\right)=\left(x, \rho_{j}(x), j \in J, \beta(x)\right)$, where $\rho_{j}(x), j \in J$, and $\beta(x)$ are defined in (6) and (11). Denote

$$
\begin{aligned}
& K_{a}(j, x):=\left\{t_{j} \in K(j): \frac{1}{2} t_{j}^{T} D_{j} t_{j}+\left(c_{j}-A_{j}^{T} x\right)^{T} t_{j}=\rho_{j}(x)\right\}, j \in J, \\
& \Delta K(j, x):=\left\{\tau \in \Delta K(j):\left(c_{j}^{T}-x^{T} A_{j}\right) \tau=0,\|\tau\|=1\right\}, j \in J .
\end{aligned}
$$

\section{Theorem 2}

A feasible solution $\left(x^{0} \in \mathbb{R}^{n}, \rho_{j}^{0}, j \in J, \beta^{0}\right)$ of the convex SIP problem (7) is optimal in this problem iff there exist vectors

$$
t_{k j}^{*} \in K_{a}\left(j, x^{0}\right), k=1, \ldots, p_{j}, \quad \tau_{k j}^{*} \in \Delta K\left(j, x^{0}\right), k=1, \ldots, l_{j}, \quad j \in J
$$

and numbers

$$
\begin{aligned}
& \lambda_{i}^{*}, i \in I ; \eta_{j}^{*}, j \in S_{*} \cup S ; \quad y_{k j}^{*}, k=1, \ldots, p_{j}, \mu_{k j}^{*}, k=1, \ldots, l_{j}, j \in J, \\
& \quad \text { with } p_{j} \geq 0, l_{j} \geq 0, j \in J, \quad \sum_{j \in J}\left(p_{j}+l_{j}\right) \leq 1+n,
\end{aligned}
$$

such that

$$
\begin{gathered}
\lambda_{i}^{*} \geq 0, \lambda_{i}^{*}\left(\frac{1}{2} x^{0^{T}} W_{i} x^{0}+d_{i}^{T} x^{0}+r_{i}-\sum_{j \in J} y_{j}^{(i)} \rho_{j}^{0}-\beta^{0}\right)=0, i \in I ; \sum_{i \in I} \lambda_{i}^{*}=1 ; \\
\eta_{j}^{*} \geq 0, \eta_{j}^{*}\left(q_{j}^{T} x^{0}+\omega_{j}\right)=0, j \in S ; \\
y_{k j}^{*} \geq 0, k=1, \ldots, p_{j}, \sum_{k=1}^{p_{j}} y_{k j}^{*}=\sum_{i \in I} \lambda_{i}^{*} y_{j}^{(i)}, \mu_{k j}^{*} \geq 0, k=1, \ldots, l_{j}, j \in J, \\
W_{0} x^{0}+d_{0}+\sum_{i \in I} \lambda_{i}^{*}\left(W_{i} x^{0}+d_{i}\right)+\sum_{j \in J} A_{j}\left(\sum_{k=1}^{p_{j}} y_{k j}^{*} t_{k j}^{*}+\sum_{k=1}^{l_{j}} \mu_{k j}^{*} \tau_{k j}^{*}\right)+\sum_{j \in S \cup S_{*}} q_{j} \eta_{j}^{*}=0 .
\end{gathered}
$$

Note that here and in what follows, we suppose that, if $\kappa=0$, then the set $\{1, \ldots, \kappa\}$ is empty and $\sum_{i=1}^{\kappa} \ldots=0$. 
Since the sets $S, S_{*}, I$ and $J$ consist of finite numbers of elements, then to simplify calculations, without loss of generality, we can present a proof of Theorem 2, considering problem (7) with $|I|=1,|J|=1$, and $\mathcal{X}=\mathbb{R}^{n}$. In other words, we will prove here the theorem for problem (7) in the following form:

$$
\begin{gathered}
\min _{x \in \mathbb{R}^{n}, \rho \in \mathbb{R}} \frac{1}{2} x^{T} W x+d^{T} x-y^{*} \rho \\
\text { s.t. } \quad \rho \leq f(t, x):=\frac{1}{2} t^{T} D t+\left(c^{T}-x^{T} A\right) t, \quad \forall t \in K=\left\{t \in \mathbb{R}^{p}: B t \leq 0\right\},
\end{gathered}
$$

where $W=W_{0}+W_{1}, d=d_{0}+d_{1}, y^{*}=y_{1}^{(1)}, K=K(1), D=D_{1}, A=A_{1}, B=B_{1}$.

Theorem 3 (A particular case of Theorem 2)

A feasible solution $\left(x^{0}, \rho^{0}\right) \in \mathbb{R}^{n+1}$ of problem (37) is optimal in this problem iff there exist vectors

$$
t_{k}^{*} \in K_{a}\left(1, x^{0}\right)=: K_{a}\left(x^{0}\right), k=1, \ldots, p_{1}, \tau_{k}^{*} \in \Delta K\left(1, x^{0}\right)=: \Delta K\left(x^{0}\right), k=1, \ldots, l_{1},
$$

and numbers

$$
y_{k}^{*}, k=1, \ldots, p_{1}, \mu_{k}^{*}, k=1, \ldots, l_{1}, \quad \text { with } p_{1} \geq 0, l_{1} \geq 0, p_{1}+l_{1} \leq 1+n,
$$

such that

$$
\begin{gathered}
y_{k}^{*} \geq 0, k=1, \ldots, p_{1}, \sum_{k=1}^{p_{1}} y_{k}^{*}=y^{*}, \mu_{k j}^{*} \geq 0, k=1, \ldots, l_{1}, \\
W x^{0}+d+A\left(\sum_{k=1}^{p_{1}} y_{k}^{*} t_{k}^{*}+\sum_{k=1}^{l_{1}} \mu_{k}^{*} \tau_{k}^{*}\right)=0 .
\end{gathered}
$$

Before proceeding with the proof, let us introduce some notation and fulfill necessary preparatory calculations.

Let $\left(x^{0}, \rho^{0}=\rho\left(x^{0}\right)\right)$ be a feasible solution of problem (37). Then the set $K_{a}\left(x^{0}\right)$ is the set of optimal solutions in the QP problem

$$
\min f\left(t, x^{0}\right) \text { s.t. } t \in K \text {. }
$$

Note that, in general, this problem is nonconvex.

It follows from [2], that the set $K_{a}\left(x^{0}\right)$ can be represented in the form $K_{a}\left(x^{0}\right)=\bigcup_{s \in \mathcal{S}} K_{s}\left(x^{0}\right)$, where for any $s \in \mathcal{S}$, the set $K_{s}\left(x^{0}\right)$ is a convex polyhedron and $|\mathcal{S}|<\infty$. Hence, for any $s \in \mathcal{S}$, there exist finite sets of (extremal) vectors $\bar{t}(s, i) \in K_{s}\left(x^{0}\right), i \in J(s)$, and rays $\bar{\tau}(s, i) \in \Delta K\left(x^{0}\right), i \in I(s)$, such that

$$
K_{s}\left(x^{0}\right)=\left\{t \in \mathbb{R}^{p}: t=\sum_{i \in J(s)} \alpha_{i} \bar{t}(s, i)+\sum_{i \in I(s)} \beta_{i} \bar{\tau}(s, i), \sum_{i \in J(s)} \alpha_{i}=1, \alpha_{i} \geq 0, i \in J(s) ; \beta_{i} \geq 0, i \in I(s)\right\} .
$$

Moreover, one can show that

$$
\begin{gathered}
\bar{t}^{T}(s, i) D \bar{t}(s, j)=\text { const, }\left(c^{T}-x^{0^{T}} A\right) \bar{t}(s, j)=\text { const }_{*}, \quad i \in J(s), j \in J(s), \\
\bar{t}^{T}(s, i) D \bar{\tau}(s, j)=0, i \in J(s), j \in I(s), \\
\bar{\tau}^{T}(s, i) D \bar{\tau}(s, j)=0, \quad \bar{\tau}(s, i) \in \Delta K\left(x^{0}\right), \quad i \in I(s), j \in I(s) .
\end{gathered}
$$

Now, consider the set $\Delta K\left(x^{0}\right)$ defined in (38). One can prove that this set is a union of a finite number of bounded convex polyhedra $\Delta K_{s}\left(x^{0}\right), s \in \Delta \mathcal{S}: \Delta K\left(x^{0}\right)=\bigcup_{s \in \Delta \mathcal{S}} \Delta K_{s}\left(x^{0}\right)$. Then, for any $s \in \Delta \mathcal{S}$, there exist finite sets of (extremal) vectors $\hat{\tau}(s, i) \in \Delta K\left(x^{0}\right), i \in \Delta I(s)$, such that

$$
\Delta K_{s}\left(x^{0}\right)=\left\{\tau \in \mathbb{R}^{p}: \tau=\sum_{i \in \Delta I(s)} \alpha_{i} \hat{\tau}(s, i), \sum_{i \in \Delta I(s)} \alpha_{i}=1, \alpha_{i} \geq 0, i \in \Delta I(s)\right\} .
$$


From the above considerations, it follows that relations

$$
-\Delta x^{T} A t \geq \Delta \rho, \forall t \in K_{a}\left(x^{0}\right), \quad-\Delta x^{T} A \tau \geq 0, \forall \tau \in \Delta K\left(x^{0}\right),
$$

are equivalent to the following ones:

$$
-\Delta x^{T} A \bar{t}(s, i) \geq \Delta \rho, i \in J(s), s \in \mathcal{S}, \quad-\Delta x^{T} A \hat{\tau}(s, i) \geq 0, i \in \Delta I(s), s \in \Delta \mathcal{S} .
$$

Here we have taken into account that $\bar{\tau}(s, i) \in \Delta K\left(x^{0}\right)$ (see (42)). Now we can prove the theorem formulated above.

\section{Proof of Theorem 3.}

Necessity. Suppose that $\left(x^{0}, \rho^{0}=\rho\left(x^{0}\right)\right)$ is an optimal solution of problem (37). Consider the following problem of Linear Programming (LP):

$$
\min _{\Delta x \in \mathbb{R}^{n}, \Delta \rho \in \mathbb{R}}\left(W x^{0}+d\right)^{T} \Delta x-y^{*} \Delta \rho \text {, s.t. conditions (44). }
$$

Suppose that the vector $\left(\Delta x^{0}, \Delta \rho^{0}\right)=0 \in \mathbb{R}^{n+1}$ is an optimal solution of this problem. Then, according to the theory of LP, there exist subsets

$$
\begin{gathered}
\left\{t_{k}^{*}, k=1, \ldots, p_{1}\right\} \subset\{\bar{t}(s, i), i \in J(s), s \in \mathcal{S}\} \subset K_{a}\left(x^{0}\right), \\
\left\{\tau_{k}^{*}, k=1, \ldots, l_{1}\right\} \subset\{\hat{\tau}(s, i), i \in \Delta I(s), s \in \Delta \mathcal{S}\} \subset \Delta K\left(x^{0}\right),
\end{gathered}
$$

and numbers (39) such that relations (40) and (41) are satisfied, and the necessity is proved.

Now, suppose that the vector $0 \in \mathbb{R}^{n+1}$ is not an optimal solution of problem (45). Then there exists a vector $(\Delta x, \Delta \rho)$, for which conditions (44) and the inequality

$$
\left(W x^{0}+d\right)^{T} \Delta x-y^{*} \Delta \rho<0
$$

are satisfied. Vector $(\Delta x, \Delta \rho)$ satisfies conditions (44), and therefore, it satisfies relations (43) as well. Consequently, according to Proposition 1 (see Appendix), for any $\delta>0$, there exists $\varepsilon_{0}=\varepsilon_{0}(\delta)>0$ such that for all $\varepsilon \in\left[0, \varepsilon_{0}\right]$, the vector $\left(x(\varepsilon):=x^{0}+\varepsilon \Delta x, \rho(\varepsilon):=\rho^{0}+\varepsilon(\Delta \rho-\delta)\right)$ is a feasible solution of problem (37).

Let us choose $0<\delta<-\left[\left(W x^{0}+d\right)^{T} \Delta x-y^{*} \Delta \rho\right] / y^{*}$ and calculate

$$
\frac{1}{2} x^{T}(\varepsilon) W x(\varepsilon)+d^{T} x(\varepsilon)-y^{*} \rho(\varepsilon)=\frac{1}{2} x^{0^{T}} W x^{0}+d^{T} x^{0}-y^{*} \rho^{0}+\varepsilon^{2} \frac{1}{2} \Delta x^{T} W \Delta x+\varepsilon \alpha_{*},
$$

where $\alpha_{*}=\left(W x^{0}+d\right)^{T} \Delta x-y^{*}(\Delta \rho-\delta)<0$. Hence, for a sufficiently small $\varepsilon>0$ we get

$$
\frac{1}{2} x^{T}(\varepsilon) W x(\varepsilon)+d^{T} x(\varepsilon)-y^{*} \rho(\varepsilon)<\frac{1}{2} x^{0^{T}} W x^{0}+d^{T} x^{0}-y^{*} \rho^{0} .
$$

But this contradicts the assumption that $\left(x^{0}, \rho^{0}=\rho\left(x^{0}\right)\right)$ is an optimal solution of problem (37). The necessity is proved.

Sufficiency. Suppose that there exist vectors (38) and numbers (39) such that relations (40) and (41) are fulfilled. Consider the convex QP problem

$$
\begin{gathered}
\min _{x \in \mathbb{R}^{n}, \rho \in \mathbb{R}} \frac{1}{2} x^{T} W x+d^{T} x-y^{*} \rho \\
\text { s.t. } \rho \leq f\left(t_{k}^{*}, x\right), k=1,2, \ldots, p_{1} ; \quad\left(c^{T}-x^{T} A\right) \tau_{k}^{*} \geq 0, k=1,2, \ldots, l_{1} .
\end{gathered}
$$

Since $\left\{t_{k}^{*}, k=1, \ldots, p_{1}\right\} \subset K_{a}\left(x^{0}\right),\left\{\tau_{k}^{*}, k=1, \ldots, l_{1}\right\} \subset \Delta K\left(x^{0}\right) \subset \Delta K$, it follows from (10) and (37) that the set of feasible solutions of problem (37) belongs to the set of feasible solutions of problem (46). Hence, vector $\left(x^{0}, \rho^{0}\right)$ is a feasible solution of problem (46) and the fulfillment of relations (40) and (41) implies that the vector is optimal in problem (46). Consequently, it is optimal in problem (37) as well. The sufficiency is proved. 
The approach that was used in the proof of Theorem 3, can be applied to the proof of Theorem 2 as well, but the calculations in this case are much more bulky. Therefore, we can consider that Theorem 2 is valid.

Observation. Let $\left(x^{0}, \rho_{j}^{0}, j \in J, \beta^{0}\right)$ be a feasible solution of problem (7). From (10), it follows that

$$
\left(c_{j}^{T}-x^{0 T} A_{j}\right) \tau_{j} \geq 0 \forall \tau_{j} \in \Delta K(j), j \in J .
$$

Suppose that the last inequalities are strongly satisfied:

$$
\left(c_{j}^{T}-x^{0^{T}} A_{j}\right) \tau_{j}>0 \forall \tau_{j} \in \Delta K(j) \backslash\{0\}, j \in J .
$$

Then $\Delta K\left(j, x^{0}\right)=\emptyset, j \in J$, and, hence, in (34)-(36) we have $l_{j}=0, \sum_{k=1}^{l_{j}} \mu_{k j}^{*} \tau_{k j}^{*}=0, j \in J, \quad\left(\right.$ or $l_{1}=0$, $\sum_{k=1}^{l_{1}} \mu_{k}^{*} \tau_{k}^{*}=0$ in (38)-(41), respectively). In this case the optimality conditions of Theorem 2 are the same as the optimality conditions formulated in $[3,5,13]$ under the assumption that problem (7) satisfies the Farkas-Minkowski CQ.

Finally, we mention two cases where the terms $\sum_{k=1}^{l_{j}} \mu_{k j}^{*} \tau_{k j}^{*}, j \in J$, can be omitted in (34)-(36):

1. $t^{T} D_{j} t>0, t \in K(j) \backslash\{0\}, j \in J$,

2. $t^{T} D_{j} t \geq 0, \forall t \in \mathbb{R}^{p}, j \in J$.

Remind that, by assumption, the inequalities $t^{T} D_{j} t \geq 0, t \in K(j), j \in J$, take place.

\section{Example}

In this section, we present an example which shows that in a general case (when condition (47) is not satisfied) of problem (7), the presence of the terms $\sum_{k=1}^{l_{j}} \mu_{k j}^{*} \tau_{k j}^{*}, j \in J$, in the optimality conditions given by relations (34)-(36) is essential.

We consider here the SIP problem in the form (37), where

$$
\begin{gathered}
n=p=2, y^{*}=1, W=\mathbb{O}_{2 \times 2}, B=A=-\mathbb{E}_{2 \times 2}, \\
c=\left(\begin{array}{l}
0 \\
0
\end{array}\right), d=\left(\begin{array}{l}
d_{1} \\
d_{2}
\end{array}\right), D_{1}=\left(\begin{array}{ll}
0 & b \\
b & 1
\end{array}\right), d_{1}>0, d_{2}>0, b>0 .
\end{gathered}
$$

Here $\mathbb{E}_{n \times n}$ denotes a $n \times n$ identity matrix.

Let us check the optimality conditions (38)-(41) of Theorem 3 (a particular case of conditions (34)-(36) of Theorem 2). Rewrite the problem in the equivalent form:

$$
\begin{gathered}
\min _{x \in \mathbb{R}^{2}, \rho} d_{1} x_{1}+d_{2} x_{2}-\rho \\
\text { s.t. } f(t, x):=\frac{1}{2} t_{2}^{2}+t_{1} t_{2} b+x_{1} t_{1}+x_{2} t_{2} \geq \rho, \forall t \in K:=\left\{t \in \mathbb{R}^{2}: t \geq 0\right\},
\end{gathered}
$$

with $d_{1}>0, d_{2}>0, b>0$, and consider the vector

$$
\left(x^{0}, \rho^{0}\right)=\left(x_{1}^{0}:=0, x_{2}^{0}:=-d_{2}, \rho^{0}:=-\frac{1}{2} d_{2}^{2}\right) .
$$


To make sure that $\left(x^{0}, \rho^{0}\right)$ is a feasible point in SIP problem (49), it is enough to notice the following:

$$
\min _{t \geq 0} f\left(t, x^{0}\right)=\min _{t \geq 0}\left(t_{1} t_{2} b+\frac{1}{2} t_{2}^{2}-d_{2} t_{2}\right)=\min _{t_{1}=0, t_{2} \geq 0}\left(\frac{1}{2} t_{2}^{2}-d_{2} t_{2}\right)=-\frac{1}{2} d_{2}^{2}=\rho^{0} .
$$

Having applied the results obtained in the previous section, one can confirm that vector $\left(x^{0}, \rho^{0}\right)$ defined in (50) is a unique optimal solution of problem (49). Indeed, in our example, we have $K_{a}\left(x^{0}\right)=\left\{t^{0}\right\}, \Delta K\left(x^{0}\right)=\left\{\tau^{0}\right\}$ with $t^{0}=\left(t_{1}^{0}=0, t_{2}^{0}=d_{2}\right), \tau^{0}=(1,0)$ and hence, according to Theorem 3, the optimality conditions (see (38)-(41)) take the form:

$$
\left(\begin{array}{c}
d_{1} \\
d_{2}
\end{array}\right)-y_{1}^{*} t_{1}^{*}-\mu_{1}^{*} \tau_{1}^{*}=0, y_{1}^{*} \geq 0, y_{1}^{*}=1, \mu_{1}^{*} \geq 0
$$

with some $t_{1}^{*} \in K_{a}\left(x^{0}\right)$ and $\tau_{1}^{*} \in \Delta K\left(x^{0}\right)$. If set $t_{1}^{*}:=t^{0}, p_{1}:=1, \tau_{1}^{*}:=\tau^{0}, l_{1}:=1, y_{1}^{*}:=1, \mu_{1}^{*}:=d_{1}>0$, and substitute in (51), we can confirm that the optimality conditions of Theorem 3 are satisfied.

Now, let us formulate for the same problem the known optimality conditions from $[5,13,3]$ without checking the fulfillment of the Farkas-Minkowski CQ. Then the optimality conditions have the form:

$$
\left(\begin{array}{c}
d_{1} \\
d_{2}
\end{array}\right)-y_{1}^{*} t_{1}^{*}=0, y_{1}^{*} \geq 0, y_{1}^{*}=1
$$

with some $t_{1}^{*} \in K_{a}\left(x^{0}\right)$. It is evident that these conditions are not fulfilled.

Note, additionally, that for problem (37) with data (48), the Farkas-Minkowski CQ is not satisfied. According to [5], the Farkas-Minkowski CQ is satisfied if the set

$$
\mathcal{K}:=\text { cone }\left\{\left(\begin{array}{c}
A t \\
1 \\
0.5 t^{T} D t+c^{T} t
\end{array}\right), t \in K ;\left(\begin{array}{l}
\mathbb{O} \\
0 \\
1
\end{array}\right)\right\}
$$

is closed. Here for $\Omega \subset \mathbb{R}^{n}$, cone $\Omega:=\left\{v=\sum_{i=1}^{n+1} \alpha_{i} \omega_{i}, \alpha_{i} \geq 0, \omega_{i} \in \Omega, i=1,2, \ldots, n+1\right\}$.

Consider the sequence of vectors and numbers

$$
t(k)=t^{0}+k \tau^{0} \in K, \alpha(k)=\frac{1}{k} ; \quad w(k)=\alpha(k)\left(\begin{array}{c}
A t(k) \\
1 \\
0.5 t^{T}(k) D t(k)+c^{T} t(k)
\end{array}\right) \in \mathcal{K}, \quad k=1,2, \ldots
$$

It is evident that there exists the limit

$$
\lim _{k \rightarrow \infty} w(k)=: w=\left(\begin{array}{c}
A \tau^{0} \\
0 \\
t^{0^{T}} D \tau^{0}
\end{array}\right)=\left(\begin{array}{c}
-\tau^{0} \\
0 \\
b d_{2}
\end{array}\right) .
$$

Let us show that $w \notin \mathcal{K}$. Suppose the contrary. Then there exist $t_{i} \in K, \alpha_{i}, i \in I$, and $\alpha_{*}$ such that

$$
\tau^{0}=\sum_{i \in I} t_{i} \alpha_{i}, \quad 0=\sum_{i \in I} \alpha_{i}, \quad b d_{2}=\sum_{i \in I} \alpha_{i}\left(0.5 t_{i}^{T} D t_{i}\right)+\alpha_{*} ; \quad \alpha_{i}>0, i \in I, \alpha_{*} \geq 0 .
$$

Evidently, the last relations are irreconcilable, that permits to conclude that the set $\mathcal{K}$ is not closed. 


\section{Conclusion}

In the paper, we considered a special class of convex SIP problems with noncompact index sets, which arise in nonlinear parametric optimization. For the problems of this class, we have formulated and proved the existence theorem and new optimality conditions.

The results of the paper will be used in study of differential properties of parametric SIP problems. Moreover, these results may be used as the basis of new approach to study of special classes of SIP problems, such as that of Copositive Programming, Semi-Infinite Polynomial Programming, and others, for which the noncompactness of index sets is commonplace.

\section{Acknowledgement}

This work was partially supported by Belarusian State Scientific Program "Convergence" and Portuguese funds through CIDMA - Center for Research and Development in Mathematics and Applications, and FCT - Portuguese Foundation for Science and Technology, within the project UID/MAT/04106/2013.

\section{REFERENCES}

1. Asprey S.P., Maccietto S. Eds. Dynamic model development: methods, theory and applications, In: Proceedings of the Workshop on the Life af a Process Model-From Conception to Action, Imperial Colledge, London, UK, October 25-26, 2000.

2. Bonnans J.F., Shapiro A. Perturbation analysis of optimization problems, Springer-Verlag, New-York, 2000.

3. Cánovas M. J., López M. A., Mordukhovich B. S., and Parra J. Variational analysis in semi-infinite and infinite programming, II: necessary optimality conditions, SIAM Journal on Optimization, vol. 20, no. 6, pp. 2788-2806, 2010.

4. Eaves B.C. On Quadratic Programming, Management Science, Theory Series, vol. 17, no. 11, pp. 698-711, 1971.

5. Goberna M.A., and López M. A. Linear Semi-Infinite Optimization, Wiley, Chichester, 1998.

6. Goberna M.A., Lópes M.A. (eds.) Semi-Infinite Programming: recent advances, Kluwer, Dordrecht, 2001.

7. Hettich R., Jongen H.Th. Semi-Infinite Programming: conditions of optimality and applications, in: J. Stoer, ed., Optimization Techniques, Part 2, Lecture Notes in Control and Information Sciences, no. 7, pp. 1-11, 1978.

8. Hettich R., Kortanek K.O. Semi-Infinite Programming: theory, methods and applications, SIAM Rev., vol. 35, pp. 380-429, 1993.

9. Hettich R., Still G. Second order optimality conditions for generalized semi-infinite programming problems, Optimization, vol. 34, pp. 195-211, 1995.

10. Kostyukova O.I., Tchemisova T.V., Kurdina M.A. A study of one class of NLP problems arising in parametric Semi-Infinite Programming, Optimization Methods and Software, Vol. 32 , Iss. 6, pp.1218-1243, 2017.

11. Kostyukova O.I., Tchemisova T.V. Implicit optimality criterion for convex SIP problem with box constrained index set, TOP, vol. 20, no. 2, pp. 475-502, 2012.

12. Kostyukova O.I., Tchemisova T.V., and Kurdina M.A. On Optimal Properties of Special Nonlinear and Semi-infinite Problems Arising in Parametric Optimization, Stat., Optim. Inf. Comput., vol. 5, pp. 99-108, 2017.

13. Mordukhovich B., Nghia T. T. A. Constraint qualifications and optimality conditions for nonconvex semi-infinite and infinite programs, Math. Program., Ser. B, vol. 139, pp. 271-300, 2013. DOI 10.1007/s10107-013-0672-x.

14. Stein O., Still G. On optimality conditions for generalized semi-infinite programming problems, J. Optim. Theory Appl., vol. 104, no. 2, pp. 443-458, 2000.

15. Wang L., Guo F. Semidefinite relaxations for semi-infinite polynomial programming, Computational Optimization and Applications, vol. 58, no. 1, pp. 133-159, 2013.

16. Weber G.-W., Kropat E., Alparslan Gök S.Z. Semi-Infinite and Conic Optimization in Modern Human Life and Financial Sciences under Uncertainty, In: ISI Proceedings of 20th Mini-EURO conference, pp. 180-185, 2008.

\section{Appendix}

\section{Proposition 1}

Assume that conditions (43) are satisfied for $(\Delta x, \Delta \rho)$. Then for any $\delta>0$, there exists $\varepsilon_{0}=\varepsilon_{0}(\delta)>0$, such that for all $\varepsilon \in\left[0, \varepsilon_{0}\right]$, vector $\left(x(\varepsilon):=x^{0}+\varepsilon \Delta x, \rho(\varepsilon):=\rho^{0}+\varepsilon(\Delta \rho-\delta)\right)$ is a feasible solution of problem (37), i.e.

$$
f(t, x(\varepsilon)) \geq \rho(\varepsilon) \forall t \in K \text {. }
$$

Proof. Rewrite relations (52) as follows:

$$
f\left(t, x^{0}\right)-\rho^{0}+\varepsilon g(t) \geq 0, \quad \forall t \in K, \quad \varepsilon \in\left[0, \varepsilon_{0}\right],
$$


where $g(t):=-\Delta x^{T} A t-\Delta \rho+\delta$.

Consider the optimization problem

$$
\varepsilon^{*}:=\inf _{t} \frac{f\left(t, x^{0}\right)-\rho^{0}}{-g(t)} \text { s.t. } t \in T:=\{t \in K: g(t) \leq 0\} .
$$

By construction, we have

$$
f\left(t, x^{0}\right)-\rho^{0}>0 \text { for all } t \in T .
$$

Hence, in (54) we have $0 \leq \varepsilon^{*} \leq \infty$. It is evident that, if $\varepsilon^{*}>0$, then relations (53) take place with $\varepsilon_{0}=\varepsilon^{*}$ and the proposition is proved in this case.

Let us show that $\varepsilon^{*} \neq 0$. Suppose that, on the contrary, $\varepsilon^{*}=0$. Let $\bar{t}^{k} \in T, k=1,2, \ldots$ be a minimizing sequence in problem (54):

$$
M_{k}:=\frac{f\left(\bar{t}^{k}, x^{0}\right)-\rho^{0}}{-g\left(\bar{t}^{k}\right)} \rightarrow 0, \quad \text { as } k \rightarrow \infty .
$$

Let us consider another sequence

$$
t^{k}=\arg \left\{\min \|t\|, \quad \text { s.t. } \frac{f\left(t, x^{0}\right)-\rho^{0}}{-g(t)} \leq M_{k}, t \in T\right\} .
$$

If suppose that the sequence $t^{k}, k=1,2, \ldots$ has a converging subsequence $t^{k_{i}}, i=1,2, \ldots$ :

$$
\lim _{i \rightarrow \infty} t^{k_{i}}=t^{*}, \lim _{i \rightarrow \infty} k_{i}=\infty
$$

then, evidently, $t^{*} \in T$ and $0=\varepsilon^{*}=\frac{f\left(t^{*}, x^{0}\right)-\rho^{0}}{-g\left(t^{*}\right)}$. But this contradicts relations (55). Thus, we can conclude that no one subsequence of the sequence $t^{k}, k=1,2, \ldots$ converges and, therefore, $\left\|t^{k}\right\| \rightarrow \infty$ as $k \rightarrow \infty$.

By assumption,

$$
0=\varepsilon^{*}=\lim _{k \rightarrow \infty} \frac{f\left(t^{k}, x^{0}\right)-\rho^{0}}{-g\left(t^{k}\right)}=\lim _{k \rightarrow \infty} \frac{\left[f\left(t^{k}, x^{0}\right)-\rho^{0}\right] /\left\|t^{k}\right\|^{2}}{-g\left(t^{k}\right) /\left\|t^{k}\right\|^{2}} \geq \frac{1}{2} \Delta \bar{\tau}^{T} D \Delta \bar{\tau},
$$

where $\Delta \bar{\tau}=\lim _{k \rightarrow \infty} t^{k} /\left\|t^{k}\right\|$. It is evident that $\Delta \bar{\tau} \in K$. Consequently, $\Delta \bar{\tau}^{T} D \Delta \bar{\tau}=0$ and $\Delta \bar{\tau} \in \Delta K$. Moreover, the inequalities $g\left(t^{k}\right)=-\Delta x^{T} A t^{k}-\Delta \rho+\delta \leq 0, k=1,2, \ldots$ imply that $-\Delta x^{T} A \Delta \bar{\tau} \leq 0$.

By assumption,

$$
0=\varepsilon^{*}=\lim _{k \rightarrow \infty} \frac{f\left(t^{k}, x^{0}\right)-\rho^{0}}{-g\left(t^{k}\right)} \geq \lim _{k \rightarrow \infty} \frac{\left[\left(c^{T}-x^{0^{T}} A\right) t^{k}-\rho^{0}\right] /\left\|t^{k}\right\|}{-g\left(t^{k}\right) /\left\|t^{k}\right\|}=\frac{\left(c^{T}-x^{0^{T}} A\right) \Delta \bar{\tau}}{\Delta x^{T} A \Delta \bar{\tau}},
$$

where $\left(c^{T}-x^{0^{T}} A\right) \Delta \bar{\tau} \geq 0$ due to (10). Hence there are two possible situations:

$$
\begin{aligned}
& \text { i) }\left(c^{T}-x^{0^{T}} A\right) \Delta \bar{\tau}=0, \Delta x^{T} A \Delta \bar{\tau}>0, \\
& \text { ii) }\left(c^{T}-x^{0^{T}} A\right) \Delta \bar{\tau}=0, \Delta x^{T} A \Delta \bar{\tau}=0 .
\end{aligned}
$$

In situation i), we have $\Delta \bar{\tau} \in \Delta K\left(x^{0}\right)$ and $\Delta x^{T} A \Delta \bar{\tau}>0$. But this contradicts relations (43).

Consider situation ii). By construction, $t^{k}=\theta^{k}\left(\Delta \bar{\tau}+\eta^{k}\right)$, where $\theta^{k}=\left\|t^{k}\right\|,\left\|\eta^{k}\right\| \rightarrow 0$ as $k \rightarrow \infty$. Note that $B\left(\Delta \bar{\tau}+\eta^{k}\right) \leq 0$. Hence, for any row $b_{m}^{T}$ of the matrix $B$, we have an implication:

$$
b_{m}^{T} \eta^{k}<0 \Rightarrow b_{m}^{T} \Delta \bar{\tau}>0 .
$$

Taking into account the last inequalities and that $\left\|\eta^{k}\right\| \rightarrow 0$ as $k \rightarrow \infty$, we conclude that $B\left(\frac{1}{2} \Delta \bar{\tau}+\eta^{k}\right) \leq 0$, for sufficiently large $k$. This implies that $\tilde{t}^{k}:=\theta^{k}\left(\frac{1}{2} \Delta \bar{\tau}+\eta^{k}\right) \in K$ for sufficiently large $k$. Rewrite the vector $\tilde{t}^{k}$ as 
follows:

$$
\tilde{t}^{k}=\theta^{k}\left(\Delta \bar{\tau}+\eta^{k}\right)-\frac{1}{2} \theta^{k} \Delta \bar{\tau}=t^{k}-\frac{1}{2} \theta^{k} \Delta \bar{\tau} .
$$

Taking into account relations (57) and the following one:

$$
t^{T} D \tau \geq 0 \text { for all } t \in K, \tau \in \Delta K,
$$

it is easy to show that

$$
f\left(\tilde{t}^{k}, x^{0}\right)=f\left(t^{k}, x^{0}\right)-\theta^{k} t^{k^{T}} D \Delta \bar{\tau} \leq f\left(t^{k}, x^{0}\right), g\left(\tilde{t}^{k}\right)=g\left(t^{k}\right), \quad\left\|\tilde{t}^{k}\right\|<\left\|t^{k}\right\| .
$$

But the last relations contradict condition (56). Hence, the situation ii) is impossible as well. Thus we can can conclude that $\varepsilon^{*}>0$. The proposition is proved. 